\title{
Commentary
}

\section{Presidential address - 2012}

\author{
Hemantha Perera ${ }^{1}$ \\ Sri Lanka Journal of Obstetrics and Gynaecology 2012; 34: 1-5
}

The aim of SLCOG as spelt out in our constitution is 'to consider matters pertaining to obstetrics and gynaecology in all its aspects, thereby strive to provide the highest possible standards to improve women's health in Sri Lanka'. As a mature professional body in existence for 58 years, the time is ripe to look back, learn and plan for the future.

We need to be responsible for the whole life cycle of a woman and also in the mechanisms and economics of looking after them. As such, the Council will be ratifying a strategic plan for the coming years, encompassing all what we have been striving to achieve over the years as well as covering new ground, with the intention of setting targets for each goal.

We believe that Sri Lanka should be looking beyond Millennium Development Goals rather than being complacent in juggling figures, meant for countries with far less developed health infrastructure.

The College has set 27 specific strategic goals to achieve in the next 05 years. These goals cover three major areas:

- Health needs in each stage of a woman's life

- Professional development in the field of obstetrics and gynaecology

- The goals we expect that the College would reach

Most importantly, majority of these goals are directed towards the better life for a woman in our country. To understand the wider responsibilities of the SLCOG, it may be worthwhile to go through the life cycle of a female.

Looking at the beginning of the "Journey of a woman", it is heartening to note that unlike some of our neighbouring countries, we have no sex

\footnotetext{
${ }^{1}$ President, Sri Lanka College of Obstetricians and Gynaecologists.

Correspondence: Hemantha Perera

E-mail: hemanthawasantha@gmail.com
}

discrimination when it comes to unborn or newborn children.

However, the real issues begin later in childhood. A girl child needs, in addition to the general education and living, protection from abuse, sexual and reproductive health education and free and easy access to sexual and reproductive health services.

\section{Why invest in children and adolescents?}

From around 10 years, a child undergoes a biological transition, which is characterised by puberty related changes in physical appearance and the attainment of reproductive capability, psychological or cognitive transition. These reflects an individual's thinking, and social transition, which is related to rights, privileges and responsibilities of an individual.

In Sri Lanka, adolescents represent 22\% of the total population. $19 \%$ of the illegal abortions taking place in the country are in the 15-25 age group. Premarital sex is not culturally accepted in Sri Lanka. But, the sexual debut for both females and males is found to be around 15 years.

This phase of life can be regarded as the last opportunity for nurturing before adulthood. Using this opportunity can contribute to prosperity while neglect will have serious repercussions on their health and wellbeing as well as an adverse effect on the national economy and development.

In 2008, the percentage of teenage pregnancies in Sri Lanka was 6.7 percent and it has been reduced to 6.5 percent in 2009, however, with marked disparities regionally. Let us teach them about these natural feelings, which may put them at a health risk at an early age and one that could permanently change their lives. Let us tell them in a way they understand and are comfortable with. But, a big BUT, if the natural feelings got the better of the teachings, then let us look after them. Surely, you would do it if it was your own child.

In a society where the words "sex education" sends shivers among conservative policy makers, it is 
imperative that a professional body such as the SLCOG, neutral from the stigma of sexually transmitted diseases or family planning, takes the leadership in gathering requisite forces to get the necessary information to the children who are the future parents of this country. I think it is a human right to know about their body and that is certainly NOT sex education.

Adolescents are eager to receive information on sexually transmitted diseases including HIV, abortions and contraception. However, they hardly get any information from service providers, as the system does not provide for it. Instead their main sources of sexual and reproductive matters are from media, friends and school.

Although some sexual and reproductive programmes targeted on the school children were carried out during the past few years, the most vulnerable population of recent school leavers is often neglected.

Has SLCOG got a role here? I think we definitely have.

We have to make the education system duty bound to provide appropriate reproductive health knowledge to make their lives safer as a right of every child from age 5 (school entry). With 100\% schooling and similar literacy rates and with computer literacy ever increasing, we have many avenues.

Can we be happy about health care services for adolescents in Sri Lanka? Availability of sexual and reproductive health services is not adequate and even the places where such facilities are available are not accessible to those who really need the services.

Youth needs places which offer advice on sexual and reproductive health issues without any stigma attached. For example, university students will not go to the health centre to seek this type of help but would be very willing if counselling trained medical students are accessible to them.

SLCOG can act as the coordinator of all stakeholders responsible for the services for this age group in a concerted plan of action towards this goal and be the leading resource of planning and technical advice of such strategy.

Let me dwell on another important issue in a woman's life in which we, the SLCOG has to come forward - contraception. It is a right of a woman to be able to prevent an unwanted pregnancy.
With only $50 \%$ of eligible couples using a satisfactory contraceptive method, we are only encouraging deaths and disease from illegal terminations. We already have the much larger effect of total fertility rate going up which has significant economic repercussions.

The country has to provide dedicated 8 am - 4 pm family planning service at every health institution. If there is no access to the services at the time the client needs, we are bound to have the repercussions of unwanted pregnancies.

Our job as members of the SLCOG is not only to attend to the medico legal and gynaecological aspects of the assault. We have to initiate processes which will ensure total physical and psychological welfare of them and also in the education of children on the preventive aspects.

It is noteworthy that SLCOG has never been represented in the National Child Protection Authority, though the issues pertaining to the girl child are clinically handled by our members. We hope the authorities will rectify this immediately.

SLCOG has initiated a training programme for the development of ultrasound scanning skills of its members and postgraduates. As such, anomaly detection should be available to the needy.

We have been collaborating with the legal authorities for appropriate amendments to the applicable laws and shall continue to canvas the government on the issue.

During the last 3 years, over 3.6 million women have been employed abroad. While the women migrant workers are responsible for the bulk of foreign investment brought into the country, the social and health repercussions to them and their children, particularly daughters, are immense. They become vulnerable prey to sexual offenders and also get into trouble due to ignorance.

Single mothers have to be recognised as a category needing special attention. The war, the lack of accessibility to sexual and reproductive health services, teenage pregnancies, increased overseas employment and social circumstances are some direct reasons for this situation. You may notice that the highest rate of suicides in females is in the 15-24 age group.

SLCOG will collaborate with the legal and labour authorities for appropriate amendments to the 
applicable laws pertaining to under aged and unmarried mothers. The government has to be persuaded for better social support for them. We also can sway the public opinion and the attitude of the health care givers via media and education.

Care before, during and after pregnancy is directly related to maternal mortality and morbidity. Preconception care is an inherent part of a healthy pregnancy. We have to encourage prospective parents to seek this service which should be available at every specialist manned hospital. With $87 \%$ of more than 4 visits to the antenatal clinic and satisfactory early registration, we have to move towards quality care now. There is a lot of room for improvement, and expertise of our College has to be utilised for this purpose. Examples are routine dating scanning, mandatory haemoglobin estimation twice and glucose tolerance test in pregnancy.

\section{Having had a remarkable reduction of maternal deaths, are we becoming complacent?}

We have been facing difficulty in reducing maternal mortality from a low level to a desirable level near zero. "No mother should die at child birth in Sri Lanka" should be our motto. With a population who are educated enough to come to the hospitals for delivery, the onus is on the health system.

I have already dealt with the key issue of unwanted pregnancies killing women. Let us examine the issue of maternal mortality more closely. Over the last two decades, the leading killers have not changed. Can we bring down the main blocks of deaths? Still direct death are leading causes and Are all mothers prepared for a possible post partum heamorrhage $(\mathrm{PPH})$ ? Is her $\mathrm{Hb}$ more than $10 \mathrm{~g} / \mathrm{dl}$ during the antenatal period? We need a minimum of two $\mathrm{Hb}$ estimations, one at booking or the first registration at an antenatal clinic and another around 32-34 weeks.

We need universal application of national partogram to prevent prolonged labour. Both these extremely basic requirements are mandatory if we are seeking a reduction of PPH mortality and morbidity. We do not need a country wide survey to realise this. Let us not redefine the wheel.

With the College members manning all hospitals, the health authorities can easily use the College to implement this as a countrywide effective programme with clinical governance principles of risk management and mandatory audits incorporated.

We need skilled staff attending the deliveries. Every now and then, birth canal trauma takes its toll.
Mandatory in-service training is a must if we were to make sure that every birth attendant, may that be a midwife, nurse or a doctor irrespective of the time of the day and the day of the week, is capable of carrying out a safe delivery.

Compulsory $\mathrm{O}$ and $\mathrm{G}$ training during internship or post intern period is a must if we are to make sure that the medical officers currently posted to the hospitals which carry out maternity care are to prevent maternal deaths.

We need early detection of postpartum haemorrhage to act during the golden hour. Implementation of a simplified guideline like the "Rule of 100" will prevent a woman going down the slippery slope before the postpartum haemorrhage is detected. Many are the tales where the initial detection has been late.

Once detected, ready access to emergency obstetrics care (EOC) can only prevent the eventual catastrophe. Take the scenario of a woman delivering in a rural or an estate hospital. Shall we say that up to the detection of the postpartum haemorrhage the staff has done well?

What next? Who will carry out the lifesaving obstetric procedures which need to be performed within minutes? And it is necessary that those be performed by the team available at the hospital rather than a "flying squad" or at specialist care hospitals after transfer, because such delays could result in a catastrophe.

My predecessor, with his vast experience in the peripheries, initiated one of the best programmes ever launched by SLCOG - the Senior House Officer Training Programme. All SHOs in active maternity care services are being trained on life saving skills among many other surgical and medical skills during a 3 day programme. All the resource personnel from the SLCOG have been wonderfully supportive in lending their time and expertise, of course without any economic compensation. We have proposed to the Ministry of Health that they immediately takeover this programme to train all medical officers, not only SHOs in specialist care hospitals, who come across women in labour on an island wide scale.

How reassuring is it to know that the medical officer in charge of a small hospital in a remote corner, few hours away from the nearest specialist care unit, is now capable of inserting a condom catheter for uterine tamponade or starting MgS04 for eclampsia before transferring the woman. We may be able to break the deadlock on PPH or PIH figures to a significant 
extent with such skills being imparted. Let us not waste millions of rupees on a needs assessment survey to find out how many of these medical officers need such training. Literally none have had training since taking up their posts and 50\% may have not managed a woman in labour after completing their medical education.

Medical disorders are the leading cause of indirect deaths. This has been the trend for some time now. While cardiac disease is the leading cause, many other medical disorders kill. When PIH also is added to this, medical disorders become the leading cause of present day maternal deaths.

The Board of Study in Obstetrics and Gynaecology has initiated a training programme in obstetric medicine. Particular emphasis has to be placed on provision of obstetric medicine via multidisciplinary teams developed within the specialist care hospitals. SLCOG will initiate this process with the support of sister colleges and associations such as Ceylon College of Physicians, Haematologists etc. with the assistance of the Ministry.

SLCOG has already distributed guidelines on the expected standards of service delivery. The Ministry has to work with us to make sure that the routine application of guidelines is practiced, based on risk management principles.

One of the drawbacks of the present system of maternal death inquiries is the lack of confidentiality which encourages a defensive attitude from the health workers. This has led to an inability to get to the root causes of deaths and thus appropriate preventive measures cannot be put in place. The SLCOG has convinced the Ministry of Health to initiate confidential enquiries with legal indemnity based on the practice followed by developed countries. In view of the low number of maternal deaths, the near deaths can teach many preventive lessons. Thus, SLCOG proposes to initiate severe acute maternal morbidity inquiries on the same basis of confidentiality.

There is an acute shortage of junior medical officers at many $\mathrm{O}$ and $\mathrm{G}$ units due to the disregard of the delivery:staff ratio. SLCOG proposes a workload based minimum staffing pyramid with immediate effect.

We also recommend to ensure continuous care by having a minimum of 02 specialists per station. It is not effective to provide intermittent services in a field such as obstetrics in which emergencies could occur out of the blue.
Particularly with the improvements of the road network, it will be much more effective to have a 24 hour specialist cover at even a longer distance than having two close by stations, both of which may be without an obstetrician over the weekend.

How are we going to develop a culture of reproductive health decision making suitable to different localities of the country? As recognised by the National Health Policy on Reproductive Matters, resource allocation should be based on local requirements. However, up to now, SLCOG has not been invited to take part in this very important decision making platform where the clinical experience of the SLCOG members including our member on the location becomes essential. Rectifying this will prevent wastage of funds and implementation of inappropriate activities. For example, spending money on a fertility clinic may not be the selected priority of the local managers of an area where anaemia and the repercussions thereof are rampant.

There are many variations of culture, health issues, compliance issues particularly related to educational standards, within our country. The regional budgets have to be sensibly allocated with local priorities taken into account. I propose that district reproductive health committees are set up with the Provincial Director of Health Services being invited to chair and the co chairs being the Regional Director of Health Services and the Hospital Obstetricians. SLCOG will impress upon the Ministry of Health the importance of a fully fledged, dedicated secondary level fertility clinic at every base hospital. This is possible with training the existing laboratory staff and we can provide the necessary expertise for such programmes.

Sub fertility affects about 1 in 8 couples. Often these patients need treatment beyond level 1 treatment. At present these services are limited to Colombo and to few other towns. Even these services are mostly provided through the private sector. There need to be better organised services at base hospitals and above where the specialists are available.

Experience and knowledge of elderly population has been used by many countries to their advantage by keeping them healthy. If not looked after, they become a burden on the society and the economy. Having recognised the national importance of utilising this window of opportunity of our elderly population, SLCOG took the step of forming an independent Menopause Society. Currently SLCOG works very closely with them and in liaison with the Health Ministry towards this goal. 
Statistics show that there is a slow but steady increase in the incidence of gynaecological cancers in the country. This should be of concern to the health authorities. Two major cancers in females, namely breast and cervix, constitute nearly $35 \%$ of all female cancers. Both these cancers are preventable and SLCOG firmly believes that it is the right of all women to be screened in order to enable prevention and early detection. The demand for this service has to be encouraged by providing the knowledge to the public through school curricula and teacher training programmes as well as the media and the web. I propose training of trainer programmes by the College for master teachers and family health workers in disseminating this knowledge. SLCOG has already initiated an organised programme in this direction which will include vaccination for cervical cancer.

By 2013 we want women victims of gender based violence to be managed in an appropriate manner safeguarding the principles of human rights. SLCOG has been working with legal and other stakeholders towards this goal. It is opportune to set up a National Women Protection Authority with statutory powers on the lines of National Child Protection Authority.

Having discussed the health needs of a woman's life, let us briefly look at the role of SLCOG in professional development. SLCOG will provide its members and other professionals in the field of obstetrics and gynaecology with the latest evidence based practice via appropriate communication tools and would encourage regular audits to ensure this goal is achieved.

SLCOG has to work much more closely with other sister organisations locally and internationally. This has been initiated with a direct dialogue with the President of the Royal College of Obstetricians and Gynaecologists, United Kingdom. We will be having many bilateral activities on education, sharing of clinical experience, etc. With our own Prof. Harsha Seneviratne heading the South Asian Federation of Obstetrics and Gynaecology (SAFOG) and our own Prof. Arulkumaran heading the International Federation of Obstetrics and Gynaecology (FIGO) from this year, we will be on a sound footing internationally for collaboration.

SLCOG will be inviting all the Presidents of the local allied professional colleges and other bodies to plan and deliver a more coordinated approach to reach our goals.
There are many governmental and non governmental organizations who are interested in the welfare of women. SLCOG wishes to act as hub for these so that repetition is avoided and efficient fund usage can be encouraged towards common goals. I sincerely request all donor agencies not to waste precious rupees or dollars on reinventing the square wheel. We have to resist attempts to bring in inappropriate global recommendations to practice 20th century medicine in Sri Lanka.

Thanks to my predecessors and the efforts by all the past and present members and well wishers, we have now got a beautiful home for the SLCOG. Our members now have a place where they are at home when in Colombo. We have one of the best auditoriums in the country. We expect closer relationships to develop among our members and their families which will make our team much stronger when working towards our goals.

Our Council will ensure that ethical standards are maintained by our members and will employ transparent remedial measures whenever the integrity of the profession is threatened. Accountability is an inherent part of $\mathrm{O}$ and $\mathrm{G}$ practice and this culture will be observed strictly.

We have always come forward for the country on our own initiative. This is what we did during the Tsunami. We went round the country including the war torn areas and attended to the affected pregnant mothers. This is what we did for internally displaced Sri Lankans during the last few days of the war. Again on our own initiative as we realised the plight of our own people as well as the possible local and international repercussions of maternal deaths during such a mammoth operation, we requested the governmemt that a task force be formed, dedicated for maternal welfare alone. Our members and the post graduate trainees covered the camps, converted a peripheral unit to an obstetrics specialist unit and made sure that a catastrophe would not occur. However, our services have not been obtained yet during the resettlement programme. It must be emphasised that adequate reproductive health services, particularly maternity services are essential to make people confident in a new settlement.

In conclusion, it may appear to you that we are embarking on an almost impossible amount of targets. But the reality is that we have been working on many of these for years and have been successful in reaching many milestones already. By introducing datelines, we expect to keep every stakeholder, especially our team in focus towards the pinnacle. 